\title{
Effects of inhaled steroids on methacholine-induced bronchoconstriction and gas trapping in mild asthma
}

\author{
A. Corsico*, R. Pellegrino**, M.C. Zoia*, L. Barbano*, V. Brusasco***, I. Cerveri*
}

Effects of inhaled steroids on methacholine-induced bronchoconstriction and gas trapping in mild asthma. A. Corsico, R. Pellegrino, M.C. Zoia, L. Barbano, V. Brusasco, I. Cerveri. (C) ERS Journals Ltd 2000.

ABSTRACT: According to a recent hypothesis, airway smooth muscle regulates airway calibre mostly at high lung volume, whereas the mucosa and adventitia dimensions dominate at low lung volumes. It was thought that if inhaled steroids decrease the thickness of airway wall in asthma, then forced vital capacity (FVC), which reflects the functional changes at low lung volume, should decrease less during induced bronchoconstriction than flow at high volume.

The study was conducted in $\mathbf{3 1}$ mild asthmatics under control conditions and during a methacholine challenge before and after 4-weeks treatment with inhaled fluticasone dipropionate (1.5 $\mathrm{mg}$ daily, 16 patients) or placebo (15 patients).

After fluticasone dipropionate treatment, control forced expiratory volume in one second (FEV1), and maximal flow at $50 \%$ of control FVC during forced expiration after a maximal $\left(V^{\prime} \max , 50\right)$ and a partial inspiration $\left(V^{\prime}, 50\right)$ significantly increased. During methacholine challenge, FVC decreased less than did FEV1 or $V^{\prime}$ max,50, and so did inspiratory vital capacity compared to $V^{\prime} \mathrm{p}, 50$. Both the provocative dose of methacholine causing a $20 \%$ fall in FEV1 and the bronchodilator effect of deep inhalation significantly increased. The latter was assessed by means of the regression coefficient of all $V^{\prime}$ max,50 plotted against $V^{\prime}, 50$. No significant changes in these parameters occurred after placebo.

These data show that inhaled steroids remarkably blunt the occurrence of gas trapping during induced bronchoconstriction in mild bronchial asthma, possibly due to their effect on airway wall remodelling.

Eur Respir J 2000; 15: 687-692.
*Clinica di Malattie dell'Apparato Respiratorio, IRCCS Policlinico S. Matteo, Pavia, **Servizio di Fisiopatologia Respiratoria, Azienda Ospedaliera S. Croce e Carle, 12100 Cuneo, and ${ }^{* * *}$ Cattedra di Fisiopatologia Respiratoria, Dipartimento di Scienze Motorie e Riabilitative, Universita di Genova, Genova, Italy.

Correspondence: I. Cerveri, Clinica di Malattie dell'Apparato Respiratorio, IRCCS Policlinico S. Matteo, Via Taramelli 5, 27100 Pavia, Italy. Fax: 390382423150

Keywords: Airway inflammation asthma

methacholine

respiratory mechanics

Received: April 281999

Accepted after revision December 151999

This study was supported, in part $(40 \%)$, by the Ministero Università Ricerca Scientifica e technologica (1997).
Airway narrowing is the crucial abnormality in bronchial asthma and may alter lung function in different ways [1-4]. The response of the asthmatic lung to asthmatic stimuli is generally inferred from changes in forced expiratory volume in one second (FEV1). It has been recently suggested that changes in forced vital capacity (FVC) may give relevant additional information as they correlate with the degree of maximal airway narrowing [4] and may reflect different pathophysiological mechanisms compared to FEV1 [5]. It has been proposed that airway smooth muscle contraction is the major determinant of the decrease in FEV1 in response to bronchoconstrictor stimuli. However, other mechanisms, such as mucosal thickening and a decreased external load on airway smooth muscle, would contribute significantly to the decrease in FVC.

Inhaled steroids represent a well-established anti-inflammatory treatment of bronchial asthma [6] and they may be expected to reduce airway wall thickening, secretions and peribronchial oedema. In this study, it was reasoned that if this were the case then inhaled steroid treatment could prevent FVC more than FEV1 from decreasing in response to bronchoconstrictor stimuli. This hypothesis was tested in 31 mild asthmatic subjects undergoing a bronchial challenge with methacholine ( $\mathrm{MCh}$ ) before and after 4-weeks treatment with inhaled fluticasone dipropionate or placebo.

Methods

\section{Subjects}

Thirty-one asthmatic subjects took part in the study (table 1). The diagnosis of asthma was based on the criteria of the American Thoracic Society [7] and its severity was defined mild according to the World Health Organization/National Heart, Lung and Blood Institute report on asthma [8]. All subjects were known to exhibit a positive response (provocative dose of methacholine causing a $20 \%$ fall in (PD20) $<1 \mathrm{mg}$ ) to an inhalation challenge with MCh. They were required to have been in stable clinical condition in the previous 2 months, to have an FEV 1 of $>80 \%$ of the predicted value and not to have suffered from upper respiratory tract infections in the previous 4 weeks. None of the subjects used antiasthmatic treatments other than short-acting $\beta_{2}$-agonists when necessary, which were avoided for $12 \mathrm{~h}$ before each study session. The study was approved by the Local Ethics 
Committee and written consent was obtained from each subject before enrolment.

\section{Study design}

This was a double-blind randomized study parallel consisting of 1-months treatment with inhaled fluticasone dipropionate $(1,500 \mu \mathrm{g}$ daily) or placebo. Lung function tests and bronchial challenge with inhaled $\mathrm{MCh}$ were performed before and after treatment.

\section{Lung function measurements}

A Vmax 22 system (SensorMedics Corporation, Yorba Linda, CA, USA) was used. Flow was measured at the mouth through a mass flow sensor, and volume obtained by numerical integration of the flow signal.

Baseline FEV1 and FVC were calculated from three reproducible maximal forced expiratory manoeuvres [9]. Then, the subjects were asked to perform three sets of reproducible manoeuvres, each one consisting of a forced expiration from $\sim 70 \%$ of FVC to residual volume (RV) (partial expiratory flow/volume curve (PEFV)) and a forced expiration from total lung capacity (TLC) (maximal expiratory flow/volume curve (MEFV)). The inspiratory vital capacity (IVC) manoeuvre preceding the MEFV was fast and no breath hold was allowed at full inflation. A single set of PEFV and MEFV was obtained during the bronchial challenge after each dose of MCh. All forced expiratory manoeuvres were preceded by $\geq 2$ min of quiet tidal breathing.

\section{MCh inhalation challenge}

Dry powder MCh (Laboratorio Farmaceutico Lofarma, Milan, Italy) was dissolved in distilled water and aerosolized using a breath-activated dosimeter system (MEFAR, Brescia, Italy) driven by compressed air $\left(1.5 \mathrm{~kg} \cdot \mathrm{m}^{-2}\right)$. The system was set to deliver $5 \mu \mathrm{L}$ solution-actuation ${ }^{-1}$. The dose of MCh was varied by changing the number of breaths, the concentration of $\mathrm{MCh}$ or both. Subjects were requested to maintain their spontaneous tidal breathing during aerosol inhalation and to refrain from taking deep breaths.

After control inhalation of saline, MCh was administered in doubling doses starting from $50 \mu \mathrm{g}$. The test was terminated when a decrement in FEV 1 of $>40 \%$ of control was attained, or upon the subject's request due to respiratory discomfort or dyspnoea.

At the end of the challenge, the subjects were given salbutamol $(200 \mu \mathrm{g}$, by metered dose inhaler) and left the laboratory after their FEV1 had returned to within $10 \%$ of the control value. No complications occurred during the challenges.

\section{Data analysis}

Expiratory flow was measured at an absolute lung volume corresponding to $50 \%$ of control FVC for both PEFV $\left(V^{\prime}, 50\right)$ and MEFV $\left(V^{\prime} \max , 50\right)$. This lung volume was determined by superimposing MEFV and PEFV at TLC, which was assumed to be constant throughout the study. IVC was calculated as the difference between the RV achieved after the PEFV and the lung volume at which the MEFV was started. Any decrement in FVC or IVC was taken as indicative of an increment in RV (gas trapping).
In order to compare the protective effects of fluticasone dipropionate against the decrease in FVC and FEV1, linear regression analysis was performed on the FVCs recorded at each step of the challenge against the corresponding FEV1 and $V^{\prime} \max , 50$. The same analysis was applied using IVCs and $V^{\prime}$, 50, which are indexes independent of the effects of deep inhalation [10]. A low regression coefficient (slope) and a high intercept indicate the occurrence of airway narrowing with a small or no increment in RV, and vice versa.

The effect of deep inhalation (DI) on airway calibre was inferred from the slope (MPslope) and intercept (MPint) of the linear regression of all $V^{\prime} \max , 50$ and $V^{\prime}$ p,50 measured during the challenge [10]. An MPslope of 0 indicates that DI fully reverses induced airway narrowing, whereas an MPslope of 1 indicates that it has no effect.

The degree of airway responsiveness was assessed by means of PD20. This was determined by interpolation between two appropriate points on the dose $(\log ) /$ response curve.

\section{Statistical analysis}

Group characteristics and baseline lung function data were compared using Student's two-tailed unpaired t-test or the Chi-squared test, as appropriate. Two-factor repeated-measure analysis of variance with Duncan's post hoc test was used to compare the effect of treatment between groups. Correlations were assessed by means of Pearson's test. A p-value of $<0.05$ was considered statistically significant. All values are reported as mean \pm SD.

\section{Results}

\section{Before treatment}

Baseline pulmonary function data (table 1) were not significantly different between groups. Airway responsiveness to MCh (PD20) and maximal doses of inhaled $\mathrm{MCh}$ were also similar in the two groups. The maximum decrements in FEV1 and FVC after MCh were $39 \pm 10 \%$ (range $20-57 \%$ ) and $29 \pm 12 \%$ (range $11-51 \%$ ) in the fluticasone group, respectively, and 40 $\pm 7 \%$ (range 30-53\%) and $27 \pm 10 \%$ (range $12-50 \%$ ) in the placebo group, respectively. The mean expiratory times of the forced expiratory manoeuvres were similar between the fluticasone dipropionate and placebo groups $(7.6 \pm 1.7$ and $7.8 \pm 2.1 \mathrm{~s}$, respectively, for the MEFV manoeuvre, and 6.4 \pm 2.5 and $6.6 \pm 1.5 \mathrm{~s}$, respectively, for the PEFV manoeuvre).

Table 1. - Subject characteristics

\begin{tabular}{lccc}
\hline & Fluticasone group & Placebo group & p-value \\
\hline Subjects n & 16 & 15 & \\
Sex M/F & $8 / 8$ & $8 / 7$ & NS \\
Age yrs & $31 \pm 9$ & $31 \pm 12$ & NS \\
Height cm & $170 \pm 12$ & $170 \pm 11$ & NS \\
Smoking n & 6 & 2 & NS \\
Atopy n & 15 & 13 & NS \\
FEV $\%$ & $98 \pm 15$ & $102 \pm 14$ & NS \\
FVC \% pred & $110 \pm 12$ & $112 \pm 12$ & NS \\
\hline
\end{tabular}

Data are presented as mean \pm SD. M: male; F: female; FEV1, forced expiratory volume in one second; FVC, forced vital capacity. 
The regression lines of FVC versus FEV1 as well as those of FVC versus $V^{\prime}$ max,50 and of IVC versus $V^{\prime}$, 50 were not significantly different (both in slope and intercept) between groups (table 2, fig. 1), suggesting similar effects of $\mathrm{MCh}$ on airway narrowing and gas trapping.

The regression line of $V^{\prime} \max , 50$ versus $V^{\prime}$,, 50 was similar in both groups, with mean MPslope values close to 1, suggesting a similar inability of the airways to dilate after DI (table 2, fig. 2) [10].

\section{After treatment}

In the fluticasone dipropionate group, but not in the placebo group, control FEV1, $V^{\prime} \max , 50$, and $V^{\prime}$ p,50 were significantly increased compared to pretreatment values (table 2).

After MCh challenge, the maximum decrements in FEV1 and FVC were $34 \pm 9 \%$ (range $20-49 \%$ ) and $19 \pm 11 \%$ (range 5-37\%), respectively, in the fluticasone dipropionate group and $39 \pm 5 \%$ (range $29-45 \%$ ) and $25 \pm 6 \%$ (range $11-35 \%$ ), respectively, in the placebo group. The maximal dose of $\mathrm{MCh}$ administered was significantly greater than that prior to treatment in the fluticasone dipropionate group but not in the placebo group. Airway responsiveness to $\mathrm{MCh}$ was significantly reduced in the fluticasone group but not in the placebo group.

The mean duration of the forced expiratory manoeuvres remained similar to that before treatment in both the fluticasone dipropionate and placebo groups $(7.7 \pm 3.0$ and $7.8 \pm$ $1.6 \mathrm{~s}$, respectively, for the MEFV manoeuvre, and $6.9 \pm 2.3$ and $7.0 \pm 1.1 \mathrm{~s}$, respectively, for the PEFV manoeuvre).

In the fluticasone dipropionate group, but not in the placebo group, the slopes of the linear regressions of $\mathrm{FVC}$ versus $\mathrm{FEV} 1, \mathrm{FVC}$ versus $V^{\prime} \max , 50$, and IVC versus $V^{\prime} \mathrm{p}, 50$ were significantly decreased and the intercepts increased (table 3, fig. 1). This indicates that, with either partial or maximal manoeuvres for any induced decrement in flow,
RV increased less after treatment with fluticasone dipropionate as compared to placebo.

MPint was increased significantly in the fluticasone dipropionate group after inhaled steroid treatment, suggesting an improved ability of DI to reverse induced bronchoconstriction (table 2, fig. 2).

The correlation coefficients of the linear regressions between relevant variables before and after fluticasone dipropionate and placebo treatment are shown in table 3 .

\section{Discussion}

The results of this study show that inhaled fluticasone dipropionate improved the lung function of mild asthmatic subjects both under control conditions and during induced bronchoconstriction.

Particularly remarkable was the greater protection afforded by fluticasone dipropionate against the increase in RV (gas trapping) than against the reduction in forced expiratory flow during the bronchial challenge.

Only subjects with mild asthma were included, which may limit the clinical relevance of the study. This was, however, done purposely in order to minimize spontaneous fluctuations as well as marked effects of fluticasone dipropionate on baseline lung function, which would have made responses to $\mathrm{MCh}$ hardly comparable. In addition, a dose of fluticasone dipropionate higher than those recommended for these patients [6] was used in order to minimize the effects of possible low adherence to therapy [11], thus increasing the likelihood of observing significant effects over a relatively short period of time. Therefore, no inference about the clinical use and dosage of inhaled steroids for regular treatment of mild asthma should be made on the basis of the recent study.

Because of nonuniform lung emptying and thoracic gas compression, expiratory flow may not decrease linearly with lung volume, especially during bronchoconstriction.

Table 2. - Functional variables before and after treatment

\begin{tabular}{|c|c|c|c|c|}
\hline & \multicolumn{2}{|c|}{ Fluticasone } & \multicolumn{2}{|c|}{ Placebo } \\
\hline & Before & After & Before & After \\
\hline FEV1 L & $3.51 \pm 0.76$ & $3.81 \pm 0.76^{\ddagger}$ & $3.65 \pm 0.83$ & $3.58 \pm 0.82$ \\
\hline FVC L & $4.65 \pm 1.03$ & $4.76 \pm 0.95$ & $4.73 \pm 1.19$ & $4.74 \pm 1.18$ \\
\hline IVC L & $4.38 \pm 0.90$ & $4.55 \pm 0.93$ & $4.42 \pm 1.02$ & $4.41 \pm 1.00$ \\
\hline$V^{\prime} \max , 50 \mathrm{~L} \cdot \mathrm{s}^{-1}$ & $3.52 \pm 1.09$ & $4.16 \pm 1.23^{\dagger}$ & $3.75 \pm 1.17$ & $3.50 \pm 1.34$ \\
\hline$V^{\prime} \mathrm{p}, 50 \mathrm{~L} \cdot \mathrm{s}^{-1}$ & $3.24 \pm 0.80$ & $4.06 \pm 1.31^{\dagger}$ & $3.30 \pm 1.26$ & $3.17 \pm 1.35$ \\
\hline log max dose of MCh mg & $6.12 \pm 1.54$ & $7.51 \pm 1.52^{+}$ & $6.50 \pm 1.21$ & $6.68 \pm 1.14$ \\
\hline \multirow{2}{*}{\multicolumn{5}{|c|}{ FVC versus $\mathrm{FEV} 1$}} \\
\hline & & & & \\
\hline Intercept & $1.50 \pm 1.11$ & $2.39 \pm 1.13 \ddagger$ & $1.63 \pm 0.95$ & $1.67 \pm 0.68$ \\
\hline Slope & $0.94 \pm 0.28$ & $0.66 \pm 0.27 \$$ & $0.87 \pm 0.26$ & $0.88 \pm 0.30$ \\
\hline \multicolumn{5}{|l|}{ FVC versus $V^{\prime} \max , 50$} \\
\hline Intercept & $3.04 \pm 1.07$ & $3.70 \pm 0.99$ & $3.22 \pm 0.95$ & $3.30 \pm 0.74$ \\
\hline Slope & $0.59 \pm 0.31$ & $0.32 \pm 0.17$ & $0.46 \pm 0.22$ & $0.49 \pm 0.29$ \\
\hline \multicolumn{5}{|l|}{ IVC versus $V_{\mathrm{p}, 50}^{\prime}$} \\
\hline Intercept & $2.86 \pm 0.97$ & $3.42 \pm 0.95 t$ & $2.91 \pm 0.76$ & $3.08 \pm 0.71$ \\
\hline Slope & $0.59 \pm 0.29$ & $0.37 \pm 0.19^{*}$ & $0.55 \pm 0.26$ & $0.57 \pm 0.45$ \\
\hline \multicolumn{5}{|l|}{$V_{\max , 50}^{\prime}$ versus $V_{\mathrm{p}, 50}^{\prime}$} \\
\hline Intercept & $0.43 \pm 0.39$ & $0.85 \pm 0.60 \dagger$ & $0.48 \pm 0.28$ & $0.51 \pm 0.29$ \\
\hline Slope & $0.99 \pm 0.28$ & $0.84 \pm 0.15$ & $1.07 \pm 0.21$ & $1.04 \pm 0.21$ \\
\hline
\end{tabular}

The Intercept and Slope are those of the linear regression analysis between the variables. $*$ : $\mathrm{p}<0.05 ; * *: \mathrm{p}<0.01 ; * * *$ : $\mathrm{p}<0.001$ versus before (Duncan test. FEV1: forced expiratory volume in one second; FVC: forced vital capacity; $V^{\prime} \max , 50$ and $V^{\prime}$ p,50: maximal and partial forced expiratory flows at $50 \%$ of control FVC, respectively; MCh: methacholine; PD20: provocative dose of methacholine causing a $20 \%$ fall in FEV1. 
a)

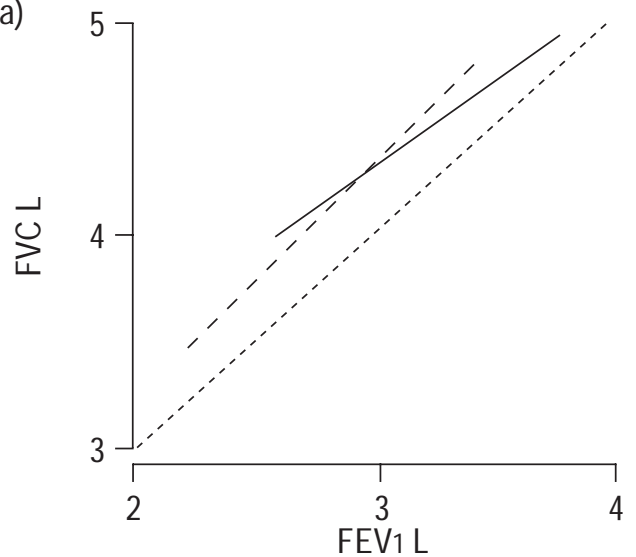

b)

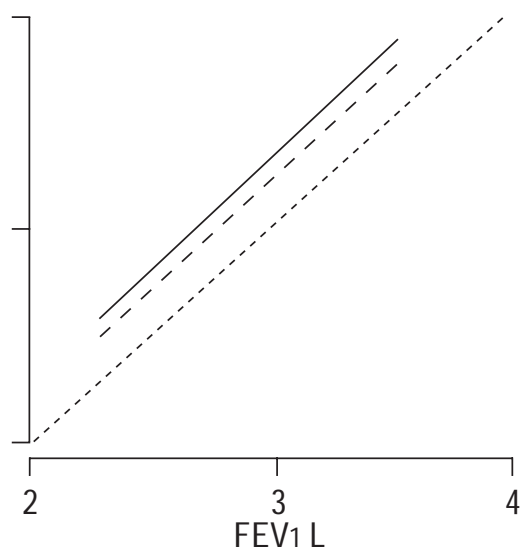

Fig. 1. - Mean regressions lines between all forced vital capacities (FVCs) and forced expiratory volume in one second (FEV1) measured during bronchial challenge with methacholine before (- - - ) and after ( - ) treatment with: a) fluticasone dipropionate; and b) placebo. Both slope and intercept were significantly different after treatment in the fluticasone dipropionate group as compared to the placebo group ( $<<0.01$ ).

High correlation coefficients between FVC and FEV1 or $V^{\prime}$ max,50 and between IVC and $V^{\prime}$ p,50 (table 3) suggest, however, that such nonlinearities do not invalidate this approach to assessing changes in the relationship between changes in flow and volume during induced bronchoconstriction.

Isovolumic measurements of $V^{\prime} \max , 50$ and $V^{\prime} \mathrm{p}, 50$ were taken by superimposing MEFVs at full inflation, thus assuming that TLC remained constant throughout the study, i.e. during both bronchial challenges and the treatment period. To the author's knowledge, TLC remains fairly constant during bronchial challenge $[12,13]$, and also after a 4-week course of inhaled steroids, as suggested by unpublished data on similar subjects from the authors' laboratory.

The bronchoconstrictor stimulus was $\mathrm{MCh}$, which is probably the most direct stimulus to airway smooth muscle, although it may also affect mucosal blood flow [14]. It cannot be excluded, therefore, that the different effects of $\mathrm{MCh}$ before and after fluticasone dipropionate might have been mediated, in part, by changes in mucosal blood flow.

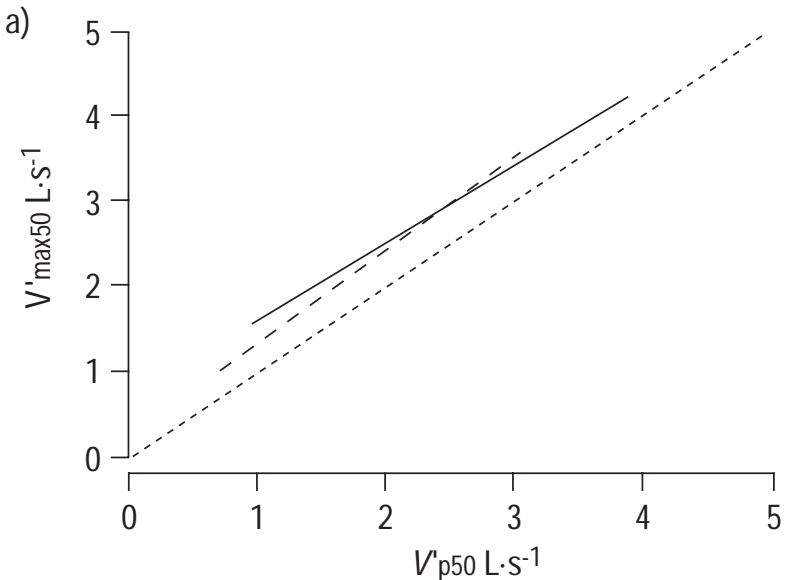

Finally, some of the subjects included in this study were smokers. As the effects of smoking may represent a confounding factor, the data from nonsmokers were reanalysed separately. All significant differences between the fluticasone dipropionate and placebo groups were still present.

Although airway smooth muscle contraction seems to be a key event in the response to bronchoconstrictor stimuli, the magnitude of airway narrowing in vivo is modulated by several factors, including mechanical load on airway smooth muscle and airway wall geometry [5, 15-17]. Major sources of mechanical load are airway wall elastic elements (internal load) and lung elastic recoil (external load). The latter is probably the most efficient mechanism opposing airway narrowing, as the response to a bronchoconstrictor stimulus is greatly reduced at increased lung volume, i.e. when lung elastic recoil is greater. The effectiveness of this mechanism in preventing airway narrowing depends on lung elastic recoil and alveolar attachments to the external airway wall $[5,15]$. Airway wall thickness seems to be the most critical geometric factor modulating

b)

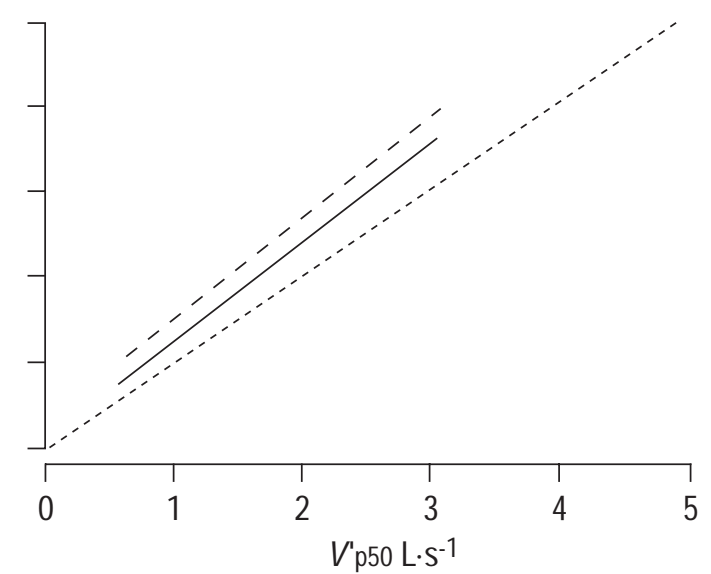

Fig. 2. - Mean regression lines between all forced expiratory flows at 50\% of control forced vital capacity for maximal ( $V^{\prime}$ max,50) and partial $\left(V^{\prime}\right.$ p,50) flow/volume curves measured during bronchial challenge with methacholine before (- - - $)$ and after $(\stackrel{-}{-})$ treatment with: a) fluticasone dipropionate; and b) placebo. The intercept was significantly different after treatment in the fluticasone dipropionate group as compared to the placebo group ( $<<0.05$ ). 
Table 3. - Correlation coefficients of the linear regressions between variables before and after fluticasone dipropionate and placebo treatment

\begin{tabular}{lcccr}
\hline & \multicolumn{2}{c}{ Fluticasone } & & \multicolumn{2}{c}{ Placebo } \\
\cline { 2 - 3 } \cline { 5 - 5 } & Before & After & Before & After \\
\hline FVC versus FEV1 & $0.98 \pm 0.02$ & $0.97 \pm 0.02$ & $0.97 \pm 0.03$ & $0.98 \pm 0.02$ \\
FVC versus $V^{\prime}$ max, 50 & $0.96 \pm 0.04$ & $0.95 \pm 0.04$ & $0.95 \pm 0.04$ & $0.96 \pm 0.04$ \\
IVC versus $V^{\prime}, 50$ & $0.95 \pm 0.04$ & $0.93 \pm 0.04$ & $0.95 \pm 0.05$ & $0.94 \pm 0.05$ \\
$V^{\prime}$ max, 50 versus $V^{\prime}$ p 50 & $0.97 \pm 0.03$ & $0.94 \pm 0.02$ & $0.94 \pm 0.02$ & $0.97 \pm 0.02$ \\
\hline
\end{tabular}

FVC: forced vital capacity; FEV1: forced expiratory volume in one second; $V^{\prime} \max , 50$ : maximal forced expiratory flow at $50 \%$ of control FVC; IVC: inspiratory vital capacity; $V^{\prime} \mathrm{p}, 50$ : partial forced expiratory flow at $50 \%$ of control FVC.

airway narrowing, as increased airway wall thickness results in an increased bronchoconstrictor response to any given degree of airway smooth muscle shortening $[15,16]$.

It is possible that inflammatory changes in the airways, such as mucosal thickening and peribronchial oedema, are more important in modulating the calibre of peripheral than of central airways [5]. In the present asthmatics, with almost normal lung function, the FEV1 reflects expiratory flow at high-to-mid lung volumes, which are probably determined by the calibre of relatively large airways, whereas the RV is probably determined by closure or extreme flow limitation of relatively smaller airways.

In the present study, inhaled fluticasone dipropionate decreased airway responsiveness to MCh (shift in PD20) but was more efficacious in preventing the increase in RV (decreases in FVC and IVC) than the decrease in FEV1, $V^{\prime} \max , 50$ and $V^{\prime} \mathrm{p}, 50$. Corticosteroids inhibit inflammatory cell migration and production of cytokines, improve antiinflammatory defences $[18,19]$, counteract fibrotic processes [20], reduce microvascular leakage [21], and, at a morphological level, they probably decrease oedema and the thickness of the wall in airways of all dimensions. A decreased thickness of the mucosa and adventitia in the small airways could preferentially prevent airway closure or extreme flow limitation. If these inferences are correct, the results of the present study would suggest that small abnormalities in the airway mucosa and adventitia may exist in subjects with mild asthma [16, 22-24] and may modulate the functional response to inhaled $\mathrm{MCh}$. This interpretation does appear to be in agreement with the increment in control FEV1 (and forced expiratory flows) not associated with changes in FVC. Possibly, in these relatively young subjects with normal or near-normal baseline lung function, the RV under control conditions was primarily regulated by the elastic properties of the chest wall [25]. Thus, even though airway calibre improved after corticosteroid therapy, the FVC could not increase accordingly. As there is no convincing evidence that the degree of airway responsiveness is related to the number of inflammatory cells in the airways [26], it is possible that the reduction in airway hyperresponsiveness after fluticasone dipropionate treatment could be related to changes in airway wall structure. By using high-resolution computed tomography, BRown et al. [27] recently examined the functional effects of bradykinin-induced airway wall oedema and found that the response to MCh was enhanced at low but not at high lung volume. This data is in keeping with the hypothesis that different mechanisms modulate airway calibre at different lung volumes.
Several studies have examined the effects of glucocorticoids on cytokine production by airway smooth muscle cells. This and other cell types, previously considered nonsecretory in nature, may be important targets for the antinflammatory effects of inhaled steroids [28]. Moreover, inhaled corticosteroids may directly modulate airway smooth muscle contractility. Even though differences in airway smooth muscle contractility have never been demonstrated between normal and asthmatic airways [29], in vitro studies have shown that allergic inflammation may alter the magnitude and velocity of airway smooth muscle shortening [30] as well as the autonomic control of airway smooth muscle tone [31, 32], which could have been affected by the fluticasone dipropionate. Other effects of corticosteroids may be a decrease in the numbers of inflammatory cells and the amount of their mediators able to enhance airway smooth muscle responsiveness [19, 20] and an increase in the number of $\beta$-adrenoceptors on airway smooth muscle cells [33]. Alternatively, the antiproliferative effect of glucocorticoids on airway smooth muscle raises the possibility that the mass of airway smooth muscle was reduced by fluticasone dipropionate treatment [28]. These direct effects of corticosteroids on airway smooth muscle cannot explain the different effects on flow and volume as they should have affected airway mechanics similarly at low and high lung volumes.

Theoretical models have shown that changing airway wall thickness or the load on airway smooth muscle markedly affects the response to bronchoconstrictor agents with only modest functional changes under control conditions [15]. This interpretation is supported by the finding that the bronchodilator effect of DI during the MCh challenge was enhanced after fluticasone dipropionate treatment, as indicated by the increase in MPint in the present study, which in turns confirms previous data by BEL et al. [34]. Corticosteroids may have improved the ability of DI to dilate constricted airways by reducing peribronchial oedema, thus restoring the forces of interdependence between the airways and the lung parenchyma, and/or by decreasing mucosal thickness, thus increasing the compliance of the wall with mechanical stretching. It should be noted, however, that the bronchodilator effect of DI during the bronchial challenge was small and remained far less than that in normal subjects [13], despite the remarkable improvement in lung function and the reduction in airway hyperresponsiveness.

In conclusion, this study sheds new light on the way in which a 4-week course of inhaled fluticasone dipropionate improves lung function in mildly asthmatic subjects. In particular, the findings show that inhaled steroids 
remarkably blunt the occurrence of gas trapping when airways narrow, probably by interfering with those structural changes in the airway wall that are a consequence of chronic inflammation. These data suggest that changes in forced vital capacity in response to bronchoconstrictor stimuli should be considered when the long-term effects of antiinflammatory treatments are to be monitored.

Acknowledgements. The authors are very grateful to J.R. Rodarte for advice and criticism, and to R. Perissin of Sensor Medics Italia (Milan, Italy) for technical assistance.

\section{References}

1. Bouhuys A, Van De Woestijne KP. Respiratory mechanics and dust exposure in byssinosis. J Clin Invest 1970; 49: $106-118$.

2. Olive JT, Hyatt RE. Maximal expiratory flow and total respiratory resistance during induced bronchoconstriction in asthmatic subjects. Am Rev Respir Dis 1972; 106: 366376.

3. Pellegrino R, Violante B, Selleri R, Brusasco V. Changes in residual volume during induced bronchoconstriction in healthy and asthmatic subjects. Am J Respir Crit Care Med 1994; 150: 363-368.

4. Gibbons WJ, Sharma A, Lougheed D, Macklem PT. Detection of excessive bronchoconstriction in asthma. $\mathrm{Am}$ J Respir Crit Care Med 1996; 153: 582-589.

5. Macklem PT. A theoretical analysis of the effect of airway smooth muscle load on airway narrowing. Am J Respir Crit Care Med 1996; 153: 83-89.

6. National Asthma Education Program. Guidelines for the diagnosis and Management of Asthma. Publication No. 91-3042. Bethesda, MD, National Institutes of Health, 1991.

7. American Thoracic Society. Chronic bronchitis, asthma and pulmonary emphysema. Am Rev Respir Dis 1962; 84: 762-768.

8. 1995. Global Initiative for Asthma. Global Strategy for Asthma Management and Prevention. NHLBI/WHO Workshop Report. Publication No. 95-3659. Bethesda, MD. National Institutes of Health.

9. American Thoracic Society. Lung function testing: selection of reference values and interpretative strategies. $\mathrm{Am}$ Rev Respir Dis 1991; 144: 1202-1218.

10. Pellegrino R, Sterk PJ, Sont JK, Brusasco V. Assessing the effect of deep inhalation on airway calibre: a novel approach to lung function in bronchial asthma and COPD. Eur Respir J 1988; 12: 1219-1227.

11. Anonymous. Are you taking the medicine? Lancet 1990; 335: 262-263.

12. Kirby JB, Juniper EF, Hargreave FE, Zamel N. Total lung capacity does not change during methacholine-stimulated airway narrowing. J Appl Physiol 1986; 61: 2144-2147.

13. Pellegrino R, Wilson O, Jenouri G, Rodarte JR. Lung mechanics during bronchoconstriction. J Appl Physiol 1996; 81: 964-975.

14. Mathe AA, Aström A, Persson NA. Some bronchoconstricting and bronchodilating responses of human isolated bronchi: evidence for the existence of $\alpha$-adrenoceptors. $J$ Pharm Pharmacol 1971; 23: 905-910.

15. Moreno RH, Hogg JC, Paré PD. Mechanics of airway narrowing. Am Rev Respir Dis 1985; 133: 1171-1180.

16. James HL, Paré PD, Hogg JC. The mechanism of airway narrowing in asthma. Am Rev Respir Dis 1989; 139: 242246.
17. Wiggs BR, Bosken C, Paré PD, James A, Hogg JC. A model of airway narrowing in asthma and in chronic obstructive pulmonary disease. Am Rev Respir Dis 1992; 145: 1251-1258.

18. Barnes PJ, Pedersen S. Efficacy and safety of inhaled corticosteroids in asthma. Report of a workshop held in Eze, France, October 1992. Am J Respir Crit Care Med 1993; 148: S1-S26.

19. Schleimer RP. Effects of glucocorticoids on inflammatory cells relevant to their therapeutic application in asthma. Am Rev Respir Dis 1990; 141: S59-S69.

20. Trigg CJ, Manolitsas ND, Wang J. Placebo-controlled immunopathologic study of four months of inhaled corticosteroids in asthma. Am J Respir Crit Care Med 1994; 150: 17-22.

21. Barnes PJ. Effects of corticosteroids on airway hyperresponsiveness. Am Rev Respir Dis 1990; 141: S70S76.

22. Djukanovic R, Roche WR, Wilson JW, et al. Mucosal inflammation in asthma. Am Rev Respir Dis 1990; 142: 434-457.

23. Kuwano K, Bosken CH, Pare PD, Bai TR, Wiggs BR, Hogg JC. Small airway dimensions in asthma and chronic obstructive lung disease. Am J Respir Crit Care Med 1993; 148: 1220-1225.

24. Carrol NG, Cooke C, James AL. Bronchial blood vessel dimensions in asthma. Am J Respir Crit Care Med 1997; 155: 689-695.

25. Leith DE, Mead J. Mechanisms determining residual volume of the lungs in normal subjects. $J$ Appl Physiol 1967; 23: 221-227.

26. Crimi E, Spanevello A, Neri M, Ind PW, Rossi GA, Brusasco V. Dissociation between airway inflammation and airway hyperresponsiveness in allergic asthma. Am J Respir Crit Care Med 1997; 157: 4-9.

27. Brown RH, Mitzner W, Wagner EM. Interaction between airway edema and lung inflation on responsiveness of individual airways in vivo. J Appl Physiol 1997; 83: 366370.

28. Hirst S, Lee TH. Airway smooth muscle as a target of glucocorticoid action in the treatment of asthma. $\mathrm{Am} \mathrm{J}$ Respir Crit Care Med 1998; 158: S201-S206.

29. Solway J, Fredberg JJ. Perhaps airway smooth muscle dysfunction contributes to asthmatic bronchial hyperresponsiveness after all. Am J Respir Cell Mol Biol 1997; 17: 144-146.

30. Mitchell RW, Ruhlmann E, Magnussen H, Leff AR, Rabe KF. Passive sensitization of human bronchi augments smooth muscle shortening velocity and capacity. $\mathrm{Am} \mathrm{J}$ Physiol 1994; 267: L218-L222.

31. Villanove X, Marthan R, Tunon de Lara JM, et al. Sensitization decreases relaxation in human isolated airway. Am J Respir Crit Care Med 1993; 148: 107-112.

32. Song P, Milanese M, Crimi E, Rehder K, Brusasco V. Allergen challenge of passively sensitized human bronchi alters $\mathrm{M}_{2}$ and $\mathrm{B}_{2}$ receptor function. Am J Respir Crit Care Med 1997; 148: 1230-1234.

33. Mak JC, Nishikawa M, Shirasaki H, Miyayasu K, Barnes PJ. Protective effects of a glucocorticoid on downregulation of pulmonary beta 2 -adrenergic receptors in vivo. J Clin Invest 1995; 96: 99-106.

34. Bel EH, Timmers MC, Hermans J, Dijkman JH, Sterk PJ. The long-term effects of nedocromil sodium and beclomethasone dipropionate on bronchial responsiveness to methacholine in nonatopic asthmatic subjects. Am Rev Respir Dis 1990; 141: 21-28. 